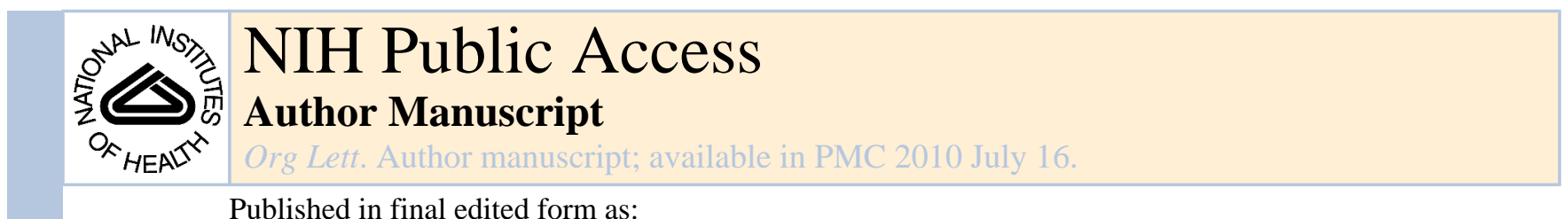

Published in final edited form as:

Org Lett. 2009 July 16; 11(14): 3108-3111. doi:10.1021/o1901096d.

\title{
Concise Synthesis of the Bryostatin A-Ring via Consecutive C-C Bond Forming Transfer Hydrogenations
}

\author{
Yu Lu and Michael J. Krische* \\ University of Texas at Austin, Department of Chemistry and Biochemistry, Austin, TX 78712
}

\section{Abstract}

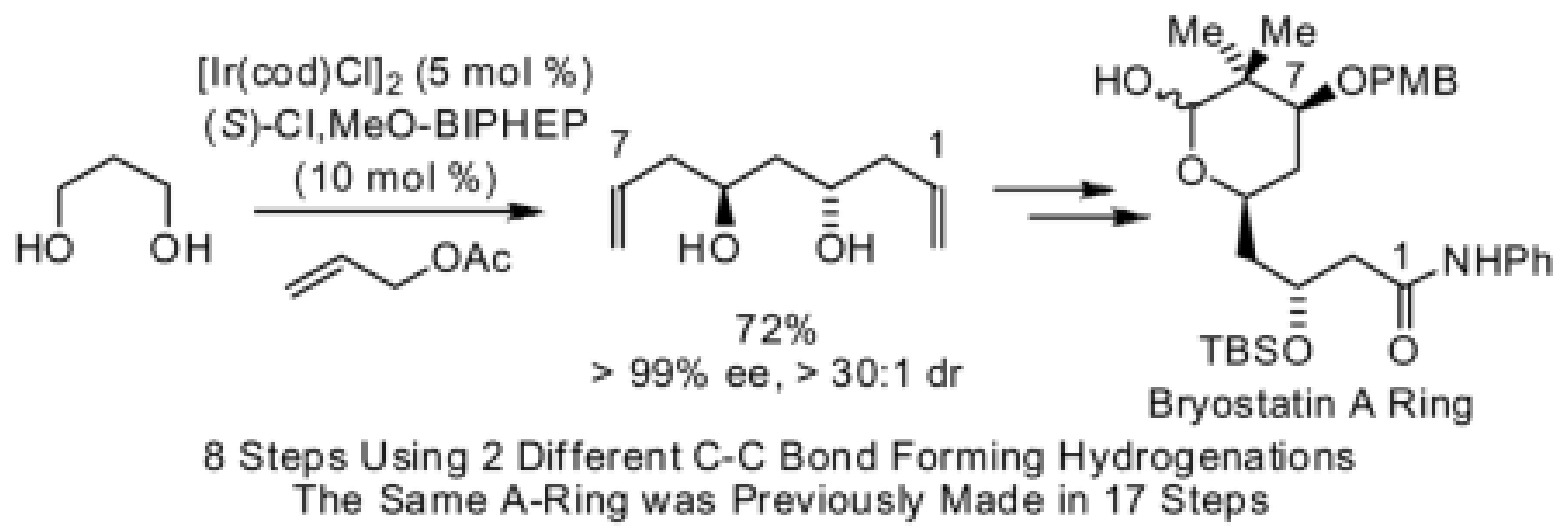

Under the conditions of $\mathrm{C}$ - $\mathrm{C}$ bond forming transfer hydrogenation, 1,3-propanediol 1 engages in double asymmetric carbonyl allylation to furnish the $C_{2}$-symmetric diol 2. Double ozonolysis of 2 followed by TBS protection delivers aldehyde 3, which is subject to catalyst directed carbonyl prenylation via transfer hydrogenation to deliver neopentyl alcohol 4 and, ultimately, the bryostatin A-ring 7. Through use of two consecutive C-C bond forming transfer hydrogenations, the Evans' bryostatin A-ring 7 is prepared in less than half the manipulations previously reported.

Originally isolated from the bryozoan Bugula neritina, ${ }^{1}$ the bryostatins are a family of over 20 marine natural products that possess a polyacetate backbone and differ largely on the basis of substitution at $\mathrm{C}_{7}$ and $\mathrm{C}_{21}$ (Figure 1, Top). ${ }^{2}$ The bryostatins exhibit a remarkable range of biological effects, including antineoplastic activity against diverse tumor cell lines, immunopotentiating activity, restoration of apoptotic function, and the ability to act synergistically with other chemotherapeutic agents. ${ }^{3}$ More recently, the bryostatins have been found to exhibit exciting neurological effects, including activity against Alzheimer's disease, ${ }^{4}$ neural growth and repair and the reversal of stroke damage, ${ }^{5}$ as well as memory enhancement. 6

The natural abundance of bryostatins is woefully insufficient to advance clinical studies and elucidate their precise mechanism of action. ${ }^{7}$ Consequently, the de novo synthesis of various bryostatins and the design and preparation of non-natural functional analogues has been the topic of intensive investigation. To date, total syntheses of bryostatin 2, bryostatin 3, bryostatin 7 and bryostatin 16 have been reported by Evans, ${ }^{8}$ Yamamura, ${ }^{9}$ Masamune, ${ }^{10}$ and Trost, ${ }^{11}$

mkrische@mail.utexas.edu.

Supporting Information Available. Spectral data for all new compounds ( ${ }^{1} \mathrm{H}$ NMR, ${ }^{13} \mathrm{C}$ NMR, IR, HRMS). This material is available free of charge via the internet at http://pubs.acs.org. 
respectively. A formal synthesis of bryostatin 7 was reported by Hale. ${ }^{12}$ Finally, several creative synthetic approaches to bryostatin fragments are reported by Hale, ${ }^{13}$ Thomas, ${ }^{14}$ Hoffman, ${ }^{15}$ Vandewalle ${ }^{16}$ Burke, ${ }^{17}$ and the present author. ${ }^{18}$ Although impressive, these syntheses do not represent a practical source of material for clinical study. Hence, efforts led by Wender, ${ }^{19,20} \mathrm{Keck}^{21}$ and Hale ${ }^{13 \mathrm{~b}}$ have focused on the design of simplified structural analogues that possess enhanced functional capability and provide insight into key structural features of the bryostatins that confer unique biological activity (Figure 1, bottom).

As part of a broad program aimed at the development of hydrogen-mediated $\mathrm{C}-\mathrm{C}$ bond formations, ${ }^{22}$ enantioselective carbonyl allylations, 23 a-c ${ }^{2}$ crotylations ${ }^{23 d}$ and reverse prenylations $^{23 \mathrm{e}}$ recently were achieved under the conditions of iridium catalyzed transfer hydrogenation. A long term goal of these studies resides in defining a departure from preformed organometallic reagents in carbonyl and imine addition, thereby circumventing the excessive pre-activation and waste generation associated with their use. Furthermore, the ability to achieve asymmetric carbonyl addition directly from the alcohol oxidation level, as established by the aforementioned processes, is inherently more redox-economic and, hence, stepeconomic than classical protocols. Given the longstanding challenges associated with defining concise routes to the bryostatins, this class of natural products was deemed an ideal vehicle to benchmark the utility of C-C bond forming hydrogenations and transfer hydrogenations developed in our laboratory. In prior work, enantioselective hydrogen-mediated alkynecarbonyl reductive coupling was applied to construction of the bryostatin C-ring. Here, using two different $\mathrm{C}-\mathrm{C}$ bond forming transfer hydrogenations, we disclose a synthetic route to a known bryostatin A-ring fragment in less than half the steps previously required. ${ }^{8}$

Our initial step toward the bryostatin A-ring employs the double asymmetric carbonyl allylation of 1,3-propanediol 1 to furnish the $C_{2}$-symmetric diol $2,{ }^{23 \mathrm{c}}$ which is obtained in > $99 \%$ ee as the minor enantiomer of the mono-adduct is converted to the meso-isomer of the bis-adduct. ${ }^{24}$ Identical material was previously prepared in four steps from acetylacetone. ${ }^{25}$ Notably, this particular carbonyl allylation cannot be achieved directly from the aldehyde oxidation level due to the instability of malondialdehyde. The $C_{2}$-symmetric diol 2 was subjected to double ozonolysis followed by protection in situ as the bis-TBS ether to deliver aldehyde 3. Because the transient dialdehyde moieties that arise upon ozonolysis are homotopic, pyran 3 appears as a single isomer (Scheme 1).

The stage was now set for catalyst-directed prenylation of aldehyde $\mathbf{3}$ under previously disclosed transfer hydrogenation conditions. ${ }^{23}$ e Upon exposure of aldehyde 3 to 1,1 dimethylallene and isopropanol in the presence of the iridium $C, O$-benzoate generated in situ from allyl acetate, $m$-nitrobenzoic acid and $(R)$-SEGPHOS, ${ }^{26}$ a $92 \%$ isolated yield of the prenylated adduct 4 was obtained as a $1: 11(S: R)$ ratio of epimers at $60{ }^{\circ} \mathrm{C}$. Using the catalyst derived from $(S)$-SEGPHOS under otherwise identical conditions, the prenylated adduct 4 was obtained as a 16:1 $(S: R)$ ratio of epimers. These data suggest that the catalysts derived from $(R)$ - and $(S)$-SEGPHOS are mismatched and matched, respectively, with regard to the inherent diastereofacial bias of aldehyde 3 . Indeed, upon use of the corresponding achiral iridium complex derived from BIPHEP, the prenylated adduct 4 was obtained as a 1.4:1 $(S: R)$ ratio of epimers corroborating a fortuitous bias of the substrate toward formation of the $(S)$-epimer. By isolating the catalyst derived from $(S)$-SEGPHOS and employing it at slightly lower temperature $\left(50{ }^{\circ} \mathrm{C}\right)$, the prenylated adduct 4 was obtained in $90 \%$ isolated yield as a single (S)-epimer as determined by ${ }^{1} \mathrm{H}$ NMR (Scheme 2).

Transformation of the neopentyl alcohol 4 to the bryostatin A-ring was accomplished in five manipulations. In accordance with literature precedent, ${ }^{27}$ the neopentyl alcohol $\mathbf{4}$ was converted to the corresponding $p$-methoxybenzyl ether in $78 \%$ isolated yield. Selective removal of the lactol TBS ether was achieved using TBAF-AcOH, also in 78\% isolated yield. 
${ }^{28}$ Finally, Jones oxidation of the lactol delivers lactone 5 in $81 \%$ isolated yield. ${ }^{29}$ Ring opening of lactone 5 mediated by $\mathrm{AlMe}_{3}-\mathrm{PhNH}_{2}$ provides anilide $\mathbf{6}$ in $84 \%$ isolated yield, ${ }^{8}$ which upon ozonolysis furnishes the previously reported bryostatin A-ring fragment $7^{8}$ in a total of eight manipulations from 1,3-propanediol 1 (Scheme 1). ${ }^{30}$ Notably, this material was prepared previously in 12 steps from 2,2-dimethyl-4,4-diphenyl-3-butenal, which itself is prepared in five steps from 3-chloro-2-methylpropene, ${ }^{31}$ thus representing a total of 17 steps from 3chloro-2-methylpropene.

To summarize, it is well-appreciated that new synthetic methods beget new synthetic strategies that can dramatically simplify the preparation of target substances. Here, through consecutive use of $\mathrm{C}-\mathrm{C}$ bond forming transfer hydrogenations, a remarkably concise synthesis of a known bryostatin A-ring fragment was achieved in less than half the manipulations previously required. In earlier work utilizing hydrogen-mediated alkyne-carbonyl reductive coupling, a route to the bryostatin C-ring was established. ${ }^{18}$ Future studies will focus on total syntheses of the bryostatins and simplified structural analogues that possess enhanced functional capability, taking advantage of the simplifications availed through $\mathrm{C}-\mathrm{C}$ bond forming hydrogenation and transfer hydrogenation.

\section{Supplementary Material}

Refer to Web version on PubMed Central for supplementary material.

\section{Acknowledgments}

Acknowledgment is made to the Robert A. Welch Foundation and the NIH-NIGMS (RO1-GM069445) for partial support of this research. Dr. Oliver Briel of Umicore is thanked for the generous donation of $[\operatorname{Ir}(\operatorname{cod}) \mathrm{Cl}]_{2}$. Takasago is thanked for the generous donation of $(S)$-SEGPHOS.

\section{References}

1. Pettit GR, Herald CL, Doubek DL, Herald DL, Arnold E, Clardy J. J Am Chem Soc 1982;104:6846.

2. (a) Pettit GR. J Nat Prod 1996;59:812. [PubMed: 8792630] (b) Lopanik N, Gustafson KR, Lindquist N. J Nat Prod 2004;67:1412. [PubMed: 15332866]

3. For selected reviews encompassing the biological properties of the bryostatins, see: (a)Hale KJ, Hummersone MG, Manaviazar S, Frigerio M. Nat Prod Rep 2002;19:413. [PubMed: 12195811](b) Kortmansky J, Schwartz GK. Cancer Invest 2003;21:924. [PubMed: 14735696](c)Wender PA, Baryza JL, Hilinski MK, Horan JC, Kan C, Verma VA. Huang Z. Beyond Natural Products: Synthetic Analogues of Bryostatin 1. In Drug Discovery Research: New Frontiers in the Post-Genomic Era Wiley-VCHHoboken, NJ2007:127-162.162

4. (a) Etcheberrigaray R, Tan M, Dewachter I, Kuiperi C, Van der Auwera I, Wera S, Qiao L, Bank B, Nelson TJ, Kozikowski AP, Van Leuven F, Alkon DL. Proc Natl Acad Sci USA 2004;101:11141. [PubMed: 15263077] (b) Alkon DL, Sun MK, Nelson TJ. Trends Pharmacol Sci 2007;28:51. [PubMed: 17218018]

5. Sun MK, Hongpaisan J, Nelson TJ, Alkon DL. Proc Natl Acad Sci USA 2008;105:13620. [PubMed: 18768786]

6. (a) Alkon DL, Epstein H, Kuzirian A, Bennett MC, Nelson TJ. Proc Natl Acad Sci USA 2005;102:16432. [PubMed: 16258064] (b) Sun MK, Alkon DL. Eur J Pharmacol 2005;512:43. [PubMed: 15814089]

7. Schaufelberger DE, Koleck MP, Beutler JA, Vatakis AM, Alvarado AB, Andrews P, Marzo LV, Muschik GM, Roach J, Ross JT, Lebherz WB, Reeves MP, Eberwein RM, Rodgers LL, Testerman RP, Snader KM, Forenza S. J Nat Prod 1991;54:1265. [PubMed: 1800630]

8. (a) Evans DA, Carter PH, Carreira EM, Prunet JA, Charette AB, Lautens M. Angew Chem Int Ed 1998;37:2354. (b) Evans DA, Carter PH, Carreira EM, Charette AB, Prunet JA, Lautens M. J Am Chem Soc 1999;121:7540. 
9. (a) Ohmori K, Ogawa Y, Obitsu T, Ishikawa Y, Nishiyama S, Yamamura S. Angew Chem Int Ed 2000;39:2290. (b) Ohmori K. Bull Chem Soc Jpn 2004;77:875.

10. Kageyama M, Tamura T, Nantz M, Roberts JC, Somfai P, Whritenour DC, Masamune S. J Am Chem Soc 1990;112:7407.

11. Trost BM, Dong G. Nature 2008;456:485. [PubMed: 19037312]

12. Manaviazar S, Frigerio M, Bhatia GS, Hummersone MG, Aliev AE, Hale KJ. Org Lett 2006;8:4477. [PubMed: 16986929]

13. (a) Review: Hale KJ, Hummersone MG, Cai J, Manavaiazar S, Bhatia GS, Lennon JA, Frigerio M, Delisser VM, Chumnongsaksarp A, Jogiya N, Lemaitre A. Pure Appl Chem 2000;72:1659.(b)Hale KJ, Frigerio M, Manaviazar S, Hummersone MG, Fillingham IJ, Barsukov IG, Damblon CF, Gescher A, Roberts GCK. Org Lett 2003;5:499. [PubMed: 12583753](c)Hale KJ, Frigerio M, Manaviazar S. Org Lett 2003;5:503. [PubMed: 12583754](d)Hale KJ, Frigerio M, Manaviazar S. Org Lett 2001;3:3791. [PubMed: 11700140](e)Hale KJ, Lennon JA, Manaviazar S, Javaid MH, Hobbs CJ. Tetrahedron Lett 1995;36:1359.(f)Hale KJ, Hummersone MG, Bhatia GS. Org Lett 2000;2:2189. [PubMed: 10930240]

14. (a) Review: Baron A, Ball M, Bradshaw B, Donnelly S, Germay O, Oller PC, Kumar N, Martin N, O'Brien M, Omori H, Moore C, Thomas EJ. Pure Appl Chem 2005;77:103.(b)Green AP, Hardy S, Thomas EJ. Synlett 2008:2103.

15. (a) Lampe TFJ, Hoffman HMR. Chem Commun 1996:1931. (b) Lampe TFJ, Hoffman HMR. Tetrahedron Lett 1996;37:7695. (c) Weiss J, Hoffman HMR. Tetrahedron: Asymmetry 1997;8:3913. (d) Vakalopoulos A, Lampe TFJ, Hoffman HMR. Org Lett 2001;3:929. [PubMed: 11263918] (e) Seidel MC, Smits R, Stark CBW, Frackenpohl J, Gaertzen O, Hoffman HMR. Synthesis 2004:1391.

16. (a) De Brabander J, Vanhessche K, Vandewalle M. Tetrahedron Lett 1991;32:2821. (b) De Brabander J, Vandewalle M. Synlett 1994:231. (c) De Brabander J, Vandewalle M. Synthesis 1994:855. (d) De Brabander J, Kulkarni A, Garcia-Lopez R, Vandewalle M. Tetrahedron: Asymmetry 1997;8:1721.

17. (a) Voight EA, Seradj H, Roethle PA, Burke SD. Org Lett 2004;6:4045. [PubMed: 15496095] (b) Voight EA, Roethle PA, Burke SD. J Org Chem 2004;69:4534. [PubMed: 15202915]

18. Cho CW, Krische MJ. Org Lett 2006;8:891. [PubMed: 16494467]

19. For recent contributions to the primary literature, see: (a)Wender PA, Verma VA. Org Lett 2006;8:1893. [PubMed: 16623578](b)Wender PA, Horan JC. Org Lett 2006;8:4581. [PubMed: 16986955](c)Wender PA, Horan JC, Verma VA. Org Lett 2006;8:5299. [PubMed: 17078702](d) Wender PA, Verma VA. Org Lett 2008;10:3331. [PubMed: 18588309](e)Wender PA, DeChristopher BA, Schreier AJ. J Am Chem Soc 2008;130:6658. [PubMed: 18452292]

20. Reviews: (a)Wender PA, Martin-Cantalejo Y, Carpenter AJ, Chiu A, De Brabander J, Harran PG, Jimenez JM, Koehler MFT, Lippa B, Morrison JA, Müller SG, Müller SN, Park CM, Shiozaki M, Siedenbiedel C, Skalitsky DJ, Tanaka M, Irie K. Pure Appl Chem 1998;70:539.(b)Wender PA, Hinkle KW, Koehler MFT, Lippa B. Med Res Rev 1999;19:388. [PubMed: 10502742](c)Wender PA, Baryza JL, Brenner SE, Clarke MO, Gamber GG, Horan JC, Jessop TC, Kan C, Pattabiraman K, Williams TJ. Pure Appl Chem 2003;75:143.(d) And reference 3c.

21. (a) Keck GE, Truong AP. Org Lett 2005;7:2153. [PubMed: 15901157] (b) Keck GE, Welch DS, Vivian PK. Org Lett 2006;8:3667. [PubMed: 16898787] (c) Keck GE, Welch DS, Poudel YB. Tetrahedron Lett 2006;47:8267. [PubMed: 17404602] (d) Keck GE, Kraft MB, Truong AP, Li W, Sanchez CC, Kedei N, Lewin NE, Blumberg PM. J Am Chem Soc 2008;130:6660. [PubMed: 18452293] (e) Keck GE, Poudel YB, Welch DS, Kraft MB, Truong AP, Stephens JC, Kedei N, Lewin NE, Blumberg PM. Org Lett 2009;11:593. [PubMed: 19113896]

22. For selected reviews on C-C bond forming hydrogenation and transfer hydrogenation, see: (a)Ngai MY, Kong JR, Krische MJ. J Org Chem 2007;72:1063. [PubMed: 17288361](b)Skucas E, Ngai MY, Komanduri V, Krische MJ. Acc Chem Res 2007;40:1394. [PubMed: 17784728](c)Shibahara F, Krische MJ. Chem Lett 2008;37:1102.(d)Bower JF, Kim IS, Patman RL, Krische MJ. Angew Chem Int Ed 2009;48:34.

23. (a) Kim IS, Ngai MY, Krische MJ. J Am Chem Soc 2008;130:6340. [PubMed: 18444616] (b) Kim IS, Ngai MY, Krische MJ. J Am Chem Soc 2008;130:14891. [PubMed: 18841896] (c) Lu Y, Kim IS, Hassan A, Del Valle DJ, Krische MJ. Angew Chem Int Ed 2009;48In Press (d) Kim IS, Han SB, Krische MJ. J Am Chem Soc 2009;131:2514. [PubMed: 19191498] (e) Han SB, Kim IS, Han H, Krische MJ. J Am Chem Soc 2009;131:6916. [PubMed: 19453190] 
24. This mechanism for enantiomeric enrichment is documented by Eliel and Midland: (a)Kogure T, Eliel ELJ. Org Chem 1984;49:576.(b)Midland MM, Gabriel J. J Org Chem 1985;50:1144.

25. Rychnovsky SD, Griesgraber G, Powers JP. Org Synth 2000;77:1.

26. SEGPHOS: Saito T, Yokozawa T, Ishizaki T, Moroi T, Sayo N, Miura T, Kumobayashi H. Adv Synth Catal 2001;343:264.

27. Wipf P, Graham TH. J Am Chem Soc 2004;126:15346. [PubMed: 15563138]

28. Orgueira HA, Bartolozzi A, Schell P, Litjens RE, Palmacci ER, Seeberger PH. Chem Eur J 2003;9:140.

29. Hung DT, Nerenberg JB, Schreiber SL. J Am Chem Soc 1996;118:11054.

30 . With regard to the assignment of relative and absolute stereochemistry, the optical rotation of $C_{2^{-}}$ symmetric diol 2 was correlated with reported data (see reference 25 and supporting information). In the reverse prenylation of 3 , the undesired diastereomer of adduct 4 obtained using $(R)$-SEGPHOS as ligand was used to complete the A-ring. As anticipated, the ${ }^{1} \mathrm{H}$ NMR data did not match the reported data. In contrast, the A-ring diastereomer obtained using $(S)$-SEGPHOS as ligand in the prenylation step matches the reported data, notwithstanding a small difference in the ratio of diastereomers at C9.

31. (a) Julia M, Baillarge M. Bull Soc Chim Fr 1966:734. (b) Zimmerman HE, Pratt AC. J Am Chem Soc 1970;92:6259. 

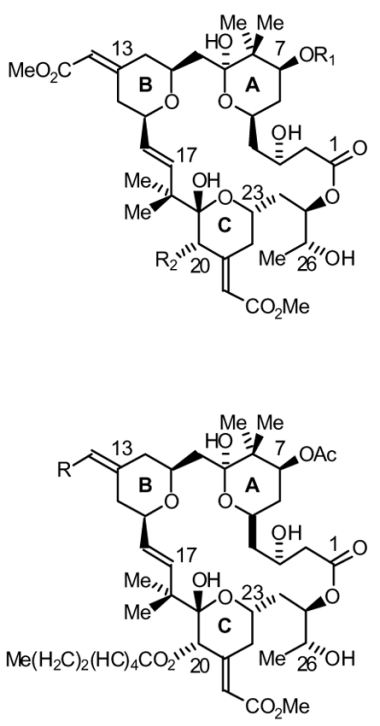

Bryostatin 1 $\mathrm{R}=\mathrm{CO}_{2} \mathrm{Me}, \mathrm{K}_{\mathrm{i}}=1.35 \mathrm{nM}$ Keck Analogue $\mathrm{A}, \mathrm{R}=\mathrm{H}, \mathrm{K}_{\mathrm{i}}=0.52 \mathrm{nM}$
Bryostatin 1, $\mathrm{R}_{1}=\mathrm{Ac}, \mathrm{R}_{2}=\mathrm{O}_{2} \mathrm{C}(\mathrm{CH})_{4}\left(\mathrm{CH}_{2}\right)_{2} \mathrm{Me}$ Bryostatin 2, $\mathrm{R}_{1}=\mathrm{H}, \mathrm{R}_{2}=\mathrm{O}_{2} \mathrm{C}(\mathrm{CH})_{4}\left(\mathrm{CH}_{2}\right)_{2} \mathrm{Me}$ Bryostatin 4, $\mathrm{R}_{1}=$ Piv, $\mathrm{R}_{2}=\mathrm{O}_{2} \mathrm{C}\left(\mathrm{CH}_{2}\right)_{2} \mathrm{Me}$ Bryostatin 5, $\mathrm{R}_{1}=$ Piv, $\mathrm{R}_{2}=\mathrm{OAC}$ Bryostatin 6, $\mathrm{R}_{1}=\mathrm{CO}\left(\mathrm{CH}_{2}\right)_{2} \mathrm{Me}, \mathrm{R}_{2}=\mathrm{OAC}$ Bryostatin 7, $\mathrm{R}_{1}=A C, \mathrm{R}_{2}=\mathrm{OAC}$ Bryostatin 8, $\mathrm{R}_{1}=\mathrm{CO}\left(\mathrm{CH}_{2}\right)_{2} \mathrm{Me}, \mathrm{R}_{2}=\mathrm{O}_{2} \mathrm{C}\left(\mathrm{CH}_{2}\right)_{2} \mathrm{Me}$ Bryostatin $9 \mathrm{R}_{1}=\mathrm{AC}_{1} \mathrm{R}_{2}=\mathrm{O}_{2} \mathrm{C}\left(\mathrm{CH}_{2}\right)_{2}$

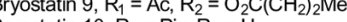
Bryostatin 10, $R_{1}=P I, R_{2}=H$

Bryostatin 12, $\mathrm{R}_{1}=\mathrm{CO}(\mathrm{CH})_{4}\left(\mathrm{CH}_{2}\right)_{2} \mathrm{Me}, \mathrm{R}_{2}=\mathrm{O}_{2} \mathrm{C}\left(\mathrm{CH}_{2}\right)_{2} \mathrm{Me}$

Bryostatin 13, $\mathrm{R}_{1}=\mathrm{CO}\left(\mathrm{CH}_{2}\right)_{2} \mathrm{Me}, \mathrm{R}_{2}=\mathrm{H}$

Bryostatin 14, $\mathrm{R}_{1}=$ Piv, $\mathrm{R}_{2}=\mathrm{OH}$

Bryostatin $15, R_{1}=A c, R_{2}=\mathrm{O}_{2} \mathrm{C}(\mathrm{CH})_{4} \mathrm{CH}(\mathrm{OH}) \mathrm{Et}$
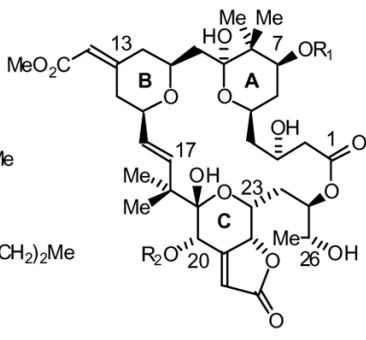

Bryostatin 3, $\mathrm{R}_{1}=\mathrm{Ac}$, $\mathrm{R}_{2}=\mathrm{O}_{2} \mathrm{C}(\mathrm{CH})_{4}\left(\mathrm{CH}_{2}\right)_{2} \mathrm{Me}$
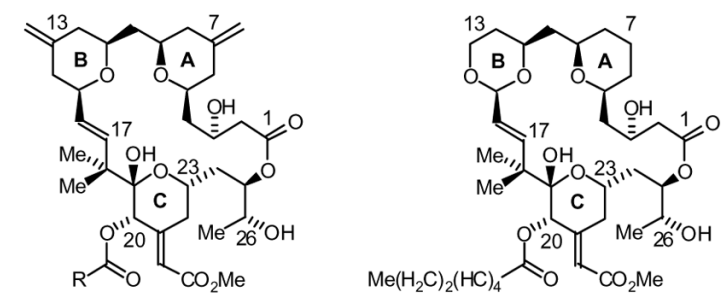

Keck Analogue $\mathrm{B}, \mathrm{R}=\mathrm{Ph}, \mathrm{K}_{\mathrm{i}}=0.70 \mathrm{nM}$ Keck Analogue $\mathrm{C}, \mathrm{R}=\mathrm{C}_{7} \mathrm{H}_{15} \mathrm{~K}=1.05 \mathrm{nM}$ Keck Analogue D, $\mathrm{R}=(\mathrm{CH})_{4}\left(\mathrm{CH}_{2}\right)_{2} \mathrm{Me}, \mathrm{K}_{\mathrm{i}}=0.70 \mathrm{nM}$

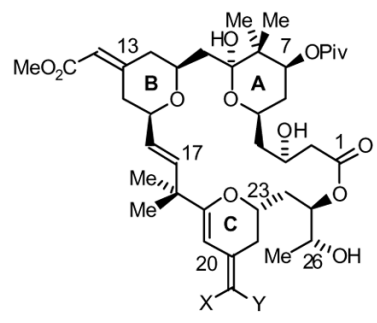

Bryostatin 16, $\mathrm{X}=\mathrm{H}, \mathrm{Y}=\mathrm{CO}_{2} \mathrm{Me}$ Bryostatin 17, $\mathrm{X}=\mathrm{CO}_{2} \mathrm{Me}, \mathrm{Y}=\mathrm{H}$

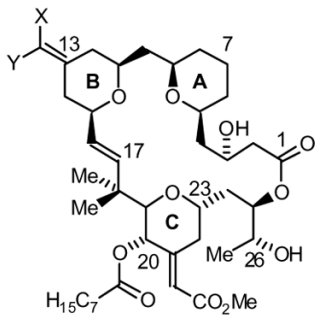

Wender Analogue $\mathrm{B}, \mathrm{X}=\mathrm{Y}=\mathrm{H}, \mathrm{K}_{\mathrm{i}}=1.6 \mathrm{nM}$ Wender Analogue $\mathrm{C}, \mathrm{X}=\mathrm{CO}_{2} \mathrm{Me}, \mathrm{Y}=\mathrm{H}, \mathrm{K}_{\mathrm{i}}=2.5 \mathrm{nM}$ Wender Analogue $\mathrm{D}, \mathrm{X}=\mathrm{H}, \mathrm{Y}=\mathrm{CO}_{2} \mathrm{Me}, \mathrm{K}_{\mathrm{i}}=0.9 \mathrm{nM}$

Figure 1.

Top: Structures of bryostatins 1-17. Bottom: Selected bryostatin analogues and binding affinities for $\mathrm{PKC} \alpha$. 

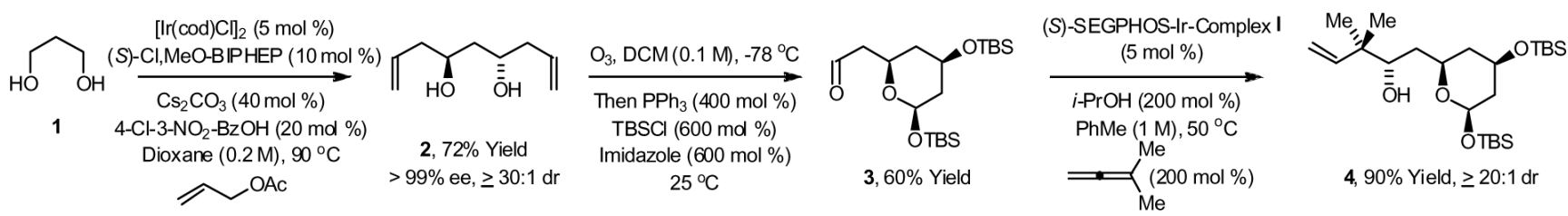

1) $\mathrm{PMBBr}(400 \mathrm{~mol} \%)$ KHMDS (400 mo \%) $\mathrm{Et}_{3} \mathrm{~N}(800 \mathrm{~mol} \%), \operatorname{THF}(0.2 \mathrm{M})$





5

Step 1, 78\% Yield

Step $2,78 \%$ Yield

Step 3, $81 \%$ Yield

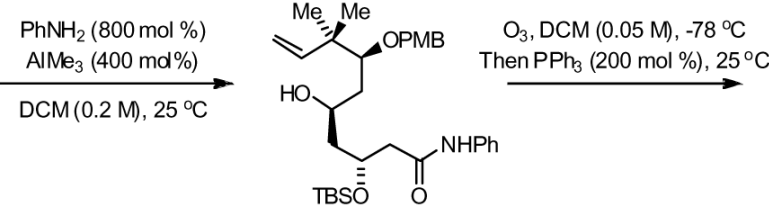

6, $84 \%$ Yield



7, $89 \%$ Yield, 1:1 dr

Scheme 1.

Synthesis of the bryostatin A-ring employing consecutive C-C bond forming transfer hydrogenations. 


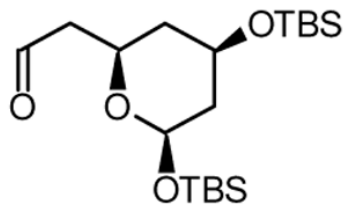

3
Ir-Complex I (5 mol \%)

$i-\operatorname{PrOH}(200 \mathrm{~mol} \%)$

PhMe (1 M), temp

$=\mathrm{Me}_{\mathrm{Me}}^{\mathrm{Me}}(200 \mathrm{~mol} \%)$

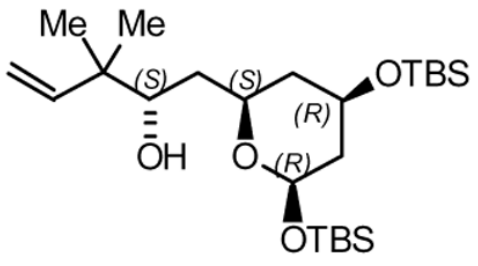

4

(R)-SEGPHOS-Ir-Complex I: 4, 92\% Yield, 1:11 dr (S:R) @ $\left.60^{\circ} \mathrm{C}\right)$ In Situ Catalyst (S)-SEGPHOS-Ir-Complex I: 4, 90\% Yield, 16:1 dr (S:R)@60 $60 \mathrm{C}\} \begin{aligned} & \text { Generation } \\ & \text { Gentu }\end{aligned}$ BIPHEP-Ir-Complex I: 4, 87\% Yield, 1.4:1 dr (S:R) @ $60{ }^{\circ} \mathrm{C}$ (S)-SEGPHOS-Ir-Complex I: 4, 90\% Yield, $\geq 20: 1 \mathrm{dr}(S: R) @ 50^{\circ} \mathrm{C} \backsim \begin{aligned} & \text { Isolated } \\ & \text { Catalyst }\end{aligned}$<smiles>Pc1cccc(-c2c(P)ccc3c2OCO3)c1-c1c(P)ccc2c1OCO2</smiles>

(S)-SEGPHOS

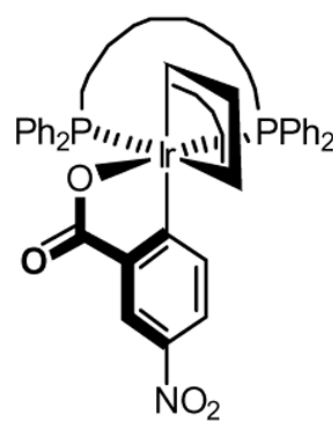

(S)-SEGPHOS-Ir-Complex I (X-Ray Diffraction, ref 23e)<smiles>Pc1ccccc1-c1ccccc1-c1ccccc1</smiles>

BIPHEP

Scheme 2.

Catalyst directed diastereoselectivity in the transfer hydrogenative reverse prenylation of aldehyde 3 . 